\title{
EFFECTS OF GROWTH HORMONE ON MUSCLE STRENGTH, TONE AND MOBILITY OF CHILDREN WITH PRADER-WILLI SYNDROME
}

\author{
S. Salehpour ${ }^{1}$, O. Aryani ${ }^{2}$
}

${ }^{1}$ Pediatric Endocrinology, Pediatric Surgery Research Center, Mofid Children's Hospital, Shaheed Beheshti University of Medical Sciences, ${ }^{2}$ Molecular Genetics, Special Medical Center, Tehran, Iran

Objective: Prader-willi syndrome (PWS) is a genetic syndrome presenting with severe hypotonia and decreased agility. Growth hormone $(\mathrm{GH})$ which is often used in these cases to treat short stature and obesity, seems to have some improvement in hypotonia, physical strength, activity, and locomotor developmental ability. The aim of this study is to find the growth hormone effect on the agility and strength of these patients.

Material \& methods: In a prospective, randomized controlled clinical trial, at an out-patient pediatric endocrine clinic in Tehran, 21 PWS children (12 boys and 9 girls, 4 to 9 years old) were divided either in GH-treated or control groups and followed for two years. Agility run, sit ups, weight lifting, and inspiratory and expiratory strengths were considered as the main outcome measures.

Results: All the outcome measures of the GH treated group showed a significant improvement compared to those of the control $(\mathrm{p}<0.01)$.

Conclusion: GH causes a significant improvement in agility and strength of PWS children. 